\title{
ANÁLISE DO DESEMPENHO DE UMA JUNTA SOLDADA PARA TUBULAÇÕES DE UMA PLATAFORMA MARÍTIMA *
}

\author{
Willy Ank de Morais ${ }^{1}$ \\ Guilherme Geada Sampaio ${ }^{2}$ \\ Rodrigo Antunes Sampaio ${ }^{3}$ \\ Leonardo Peres de Souza ${ }^{4}$
}

\section{Resumo}

Este trabalho analisou o caso de emprego de um consumível constituído de aço carbono (ER 70S3) para realizar a soldagem de tubos ASTM A333, empregados em baixas temperaturas, ao invés de empregar consumíveis especiais especificados para emprego em baixas temperaturas, tal como o ER 80S Ni2. Os três pontos centrais considerados neste trabalho foram: (1) compatibilidade entre os materiais envolvidos; (2) análise das características específicas do aço, no grau empregado e do projeto da tubulação e; (3) longevidade da linha, que pode ser prejudicada a longo prazo pelas variações químicas dos metais envolvidos que poderiam induzir corrosão localizada.

Palavras-chave: Soldagem; Aço carbono; Baixas temperaturas; Tubulações; Plataformas marítimas.

\section{PERFORMANCE ANALYSIS OF A WELDING JOINT OF A PIPELINE ON AN OFFSHORE PLATFORM}

\begin{abstract}
This work examined the case of employing a filler metal consisting of carbon steel (ER 70S3) to realize the welding of ASTM A333 tubes, used at low temperatures, instead of using special consumables specified to low temperatures work, such as $\mathrm{Ni} 2 \mathrm{ER}$ 80S. The three main points considered in this study were: (1) compatibility between the materials involved; (2) analysis of the specific characteristics of steel, employed grade and pipeline specification and; (3) longevity of the line, which can be damaged by long-term chemical variations of the metals used that could induce pitting corrosion.
\end{abstract}

Keywords: Welding; Carbon Steel; Low temperature; Pipeline; Off-Shore platforms.

1 Doutorando, Mestre, Engenheiro e Técnico em Metalurgia e Materiais, Sócio-Diretor da Willy Ank Soluções Metal-Mecânicas, Professor Mestre da UNAERP-Guarujá e Professor Adjunto da Universidade Santa Cecília (UNISANTA), Santos, SP, Brasil (willyank@unisanta.br)..

2 Técnico em Metalurgia, Inspetor de Soldagem Nível 2 (ASME B31.1), Diretor Comercial, INSPEBRAS, Praia Grande, SP, Brasil (guilherme@inspebras.com.br).

3 Técnico em Metalurgia, Inspetor de Soldagem Nível 2 (AWS), Diretor Técnico, INSPEBRAS, Praia Grande, SP, Brasil (rodrigo@inspebras.com.br).

4 Engenheiro de Inspeção de Equipamentos da German Engenharia, mestrando em Engenharia Mecânica, Universidade Santa Cecília, Santos, Brasil (leonardo.peres.eng@hotmail.com). 


\section{INTRODUÇÃO}

O sucesso da soldagem está associado a diversos fatores e, em particular, com a sua relativa simplicidade e flexibilidade operacional [1]. Por outro lado, não pode ser esquecido que a soldagem muitas vezes torna-se um processo traumático para o material, envolvendo, em geral, a aplicação de uma elevada densidade de energia em um pequeno volume do material, o que pode levar a importantes alterações estruturais e de propriedades dentro e próximo da região da solda [2].

O desconhecimento ou a simples desconsideração das implicações desta característica fundamental pode resultar em problemas inesperados e, em alguns casos, graves [3]. Estes problemas podem se refletir tanto em atrasos na fabricação ou em gastos inesperados, quando o problema é prontamente detectado, ou mesmo em perdas materiais e, eventualmente, de vidas, quando o problema é levado às suas últimas consequências.

Todo o processo de união de metais, através de componentes mecânicos ou componentes estruturais, através da técnica de soldagem tem que ser avaliado integralmente para a verificação e comprovação do desempenho do novo componente. Particularmente, para especificar corretamente 0 processo e 0 consumível de solda mais adequado é necessário conhecer o tipo de material que será soldado. Neste contexto, deve-se considerar que o princípio básico da soldagem é gerar uma união que apresente características químicas, físicas, mecânicas e geométricas as mais próximas o possível dos materiais e estruturas que estão sendo unidos para garantir sua confiabilidade [1 e 3].

O presente trabalho analisa a real necessidade do emprego de consumíveis especiais, tal como o ER $80 \mathrm{~S} \mathrm{Ni2} \mathrm{[4],} \mathrm{na} \mathrm{soldagem} \mathrm{de} \mathrm{tubos} \mathrm{de} \mathrm{aço} \mathrm{carbono} \mathrm{ASTM}$ A333 Gr.6 [5]. Neste caso, os tubos, especificados por norma [5] para atuar em temperaturas até $-45^{\circ} \mathrm{C}$, estão sendo empregados em uma linha projetada para trabalhar com fluídos a $0^{\circ} \mathrm{C}$, atingindo possíveis picos de $-20^{\circ} \mathrm{C}$. Apesar do consumível ER 80S Ni2 [4] ser especificado para uso em baixas temperaturas, este possui uma composição química e características técnicas que não recomendariam o seu emprego na tubulação estudada, que é empregada em uma plataforma offshore.

\section{MATERIAIS E MÉTODOS}

A correta definição do tipo de consumível de solda a ser empregado requer uma correta descrição dos materiais envolvidos, conforme descrito a seguir.

\subsection{Descrição do Material dos Tubos}

A norma aplicável para os tubos analisados é a ASTM A333 (Standard Specification for Seamless and Welded Steel Pipe for Low-Temperature Service and Other Applications with Required Notch Toughness) [5]. Esta norma especifica características químicas e mecânicas para tubos sem costura ou com costura (produzidos a partir de chapas conformadas e soldadas) de aço carbono ou aço baixa liga destinado a aplicações em baixas temperaturas (entre $-45 \mathrm{a}-195^{\circ} \mathrm{C}$ ).

\subsubsection{Composição química}


A norma ASTM A333 [5] especifica 8 graus de aço em termos de composições químicas. Conforme descrito pela Tabela 1, reproduzida diretamente da norma. A ASTM A333 [5] não exige a adição de elementos de liga especiais (além do carbono, manganês e silício) para os graus 1, 6 e 10. Além disso a norma ASTM A333 [5] faz a seguinte exigência, conforme descrita no item 6 , para os graus nos quais não se prevê adição de Níquel e de outros elementos de liga especiais:

"When grades 1, 6, or 10 are ordered under this specification, supplying na alloy grade that specifically requires the addition of any elemento other than those listed for the ordered grade in Table 1 is not permitted. However, the addition of elements required for the deoxidation of the steel is permitted."

Esta passagem deixa explícito que não podem ser empregados outros elementos além daqueles cujos limites foram citados na tabela de composições químicas desta norma (Tabela 1). Ou seja, a norma não permite que sejam adicionados os elementos Níquel, Cromo, Cobre, Vanádio, Nióbio, Molibdênio e Cobalto aos aços nos graus 1, 6 e 10, empregados para produzir tubos. A exceção seria para os elementos Alumínio e Silício, reconhecidamente empregados pelas siderúrgicas para a desoxidação dos aços, ainda no estado líquido [6]. Para os demais graus, a norma exige pelo ao menos a adição de Níquel, além de outros elementos que devem estar presentes além do Níquel, em função do grau considerado da norma.

Tabela 1. Composições químicas exigidas para alguns graus da norma ASTM A333 [5].

\begin{tabular}{|c|c|c|c|c|c|c|c|c|c|}
\hline \multirow{2}{*}{ Element } & \multicolumn{9}{|c|}{ Composition, \% } \\
\hline & Grade $1^{A}$ & Grade 3 & Grade 4 & Grade $6^{A}$ & Grade 7 & Grade 8 & Grade 9 & Grade 10 & Grade 11 \\
\hline Carbon, max & 0.30 & 0.19 & 0.12 & 0.30 & 0.19 & 0.13 & 0.20 & 0.20 & 0.10 \\
\hline Manganese & $0.40-1.06$ & $0.31-0.64$ & $0.50-1.05$ & $0.29-1.06$ & $0.90 \max$ & $0.90 \max$ & $0.40-1.06$ & $1.15-1.50$ & $0.60 \max$ \\
\hline Phosphorus, max & 0.025 & 0.025 & 0.025 & 0.025 & 0.025 & 0.025 & 0.025 & 0.035 & 0.025 \\
\hline Sulfur, $\max$ & 0.025 & 0.025 & 0.025 & 0.025 & 0.025 & 0.025 & 0.025 & 0.015 & 0.025 \\
\hline Silicon & $\ldots$ & $0.18-0.37$ & $0.08-0.37$ & $0.10 \mathrm{~min}$ & $0.13-0.32$ & $0.13-0.32$ & ... & $0.10-0.35$ & $0.35 \max$ \\
\hline Nickel & $\ldots$ & $3.18-3.82$ & $0.47-0.98$ & $\ldots$ & $2.03-2.57$ & $8.40-9.60$ & $1.60-2.24$ & $0.25 \max$ & $35.0-37.0$ \\
\hline Chromium & $\ldots$ & ... & $0.44-1.01$ & $\ldots$ & $\ldots$ & $\ldots$ & $\ldots$ & $0.15 \max$ & $0.50 \max$ \\
\hline Copper & $\ldots$ & $\ldots$ & $0.40-0.75$ & $\ldots$ & $\ldots$ & $\ldots$ & $0.75-1.25$ & $0.15 \max$ & $\ldots$ \\
\hline Aluminum & $\ldots$ & $\ldots$ & $0.04-0.30$ & $\ldots$ & $\cdots$ & $\ldots$ & ... & $0.06 \max$ & $\ldots$ \\
\hline Vanadium, max & $\ldots$ & $\ldots$ & $\ldots$ & $\ldots$ & $\ldots$ & $\ldots$ & $\ldots$ & 0.12 & $\ldots$ \\
\hline Columbium, max & $\ldots$ & $\ldots$ & $\ldots$ & $\ldots$ & $\ldots$ & $\ldots$ & $\ldots$ & 0.05 & $\ldots$ \\
\hline Molybdenum, max & ... & $\cdots$ & $\cdots$ & $\cdots$ & $\ldots$ & $\ldots$ & $\ldots$ & 0.05 & $0.50 \max$ \\
\hline Cobalt & $\ldots$ & $\ldots$ & ... & $\ldots$ & $\ldots$ & $\ldots$ & $\ldots$ & $\ldots$ & $0.50 \max$ \\
\hline
\end{tabular}

Assim sendo, pode-se concluir que os tubos de aço que atendam a norma ASTM A333 [5], no grau 6 não devem possuir elementos de liga especiais presentes, especialmente Níquel. Adicionalmente, outra norma da mesma normatizadora, a ASTM A1040 [7], informa qual é o teor de níquel que deve ser considerado como o teor máximo presente deste elemento: $0,3 \% \mathrm{Ni}$. Assim sendo, pode-se afirmar que os tubos de aço que atendem a norma ASTM A333 Gr. 6 [5] não devem ter um teor de Níquel acima de 0,3\%Ni [7].

\subsubsection{Propriedades Mecânicas}

A norma ASTM A333 [5] descreve os requisitos mecânicos que devem ser atendidos pelos tubos em cada grau definido pela norma. Os valores de limite de escoamento (SLE), limite de resistência $\left(S_{L R}\right)$ e alongamento que devem ser atendidos são descritos por uma tabela nesta norma. Os valores para o limite de escoamento (SLE $\geq 240 \mathrm{MPa}$ ) e limite de resistência $\left(S_{\mathrm{LR}} \geq 415 \mathrm{MPa}\right)$ a serem atendidos pelos tubos classificados no grau 6 desta norma estão apresentados na Tabela 2. Os valores de 
resistência mecânica para o grau 6 da ASTM A333A [5] são característicos de um aço de baixa resistência conforme a ABNT ( $S_{L E} \leq 280 \mathrm{MPa}$ ) [6] e pela própria ASTM $\left(S_{L E} \leq 275 \mathrm{MPa}\right)[7]$.

Tabela 2. Propriedades mecânicas obtidas em ensaios de tração que devem ser atendidas por alguns graus da norma ASTM A333 [5].

\begin{tabular}{|c|c|c|c|c|c|c|c|c|c|c|c|c|c|c|c|c|c|c|}
\hline \multirow[b]{2}{*}{$\quad \quad$} & \multicolumn{2}{|c|}{ Grade 1} & \multicolumn{2}{|c|}{ Grade 3} & \multicolumn{2}{|c|}{ Grade 4} & \multicolumn{2}{|c|}{ Grade 6} & \multicolumn{2}{|c|}{ Grade 7} & \multicolumn{2}{|c|}{ Grade 8} & \multicolumn{2}{|c|}{ Grade 9} & \multicolumn{2}{|c|}{ Grade 10} & \multicolumn{2}{|c|}{ Grade 11} \\
\hline & psi & $\mathrm{MPa}$ & psi & $\mathrm{MPa}$ & psi & $\mathrm{MPa}$ & psi & $\mathrm{MPa}$ & psi & $\mathrm{MPa}$ & psi & $\mathrm{MPa}$ & psi & $\mathrm{MPa}$ & psi & $\mathrm{MPa}$ & psi & $\mathrm{MPa}$ \\
\hline $\begin{array}{l}\text { Tensile strength, } \min \ldots \\
\text { Yield strength, } \min \ldots\end{array}$ & $\left|\begin{array}{ll}55 & 000 \\
30 & 000\end{array}\right|$ & $\begin{array}{l}380 \\
205 \\
\end{array}$ & $\left|\begin{array}{ll}65 & 000 \\
35 & 000\end{array}\right|$ & $\begin{array}{l}450 \\
240\end{array}$ & $\left|\begin{array}{ll}60 & 000 \\
35 & 000\end{array}\right|$ & $\begin{array}{l}415 \\
240\end{array}$ & $\left|\begin{array}{ll}60 & 000 \\
35 & 000\end{array}\right|$ & $\begin{array}{l}415 \\
240 \\
\end{array}$ & $\left|\begin{array}{ll}65 & 000 \\
35 & 000\end{array}\right|$ & $\begin{array}{l}450 \\
240\end{array} \mid$ & $\begin{array}{ll}100 & 000 \\
75 & 000\end{array}$ & $\begin{array}{l}690 \\
515\end{array}$ & $\left|\begin{array}{ll}63 & 000 \\
46 & 000\end{array}\right|$ & \begin{tabular}{|l|}
435 \\
315 \\
\end{tabular} & $\left|\begin{array}{ll}80 & 000 \\
65 & 000\end{array}\right|$ & $\begin{array}{l}550 \\
450 \\
\end{array}$ & $\left|\begin{array}{ll}65 & 000 \\
35 & 000\end{array}\right|$ & $\begin{array}{l}450 \\
240 \\
\end{array}$ \\
\hline : & \begin{tabular}{|l|} 
Longi- \\
tudinal
\end{tabular} & \begin{tabular}{|c|} 
Trans- \\
verse
\end{tabular} \mid & | Longi- $\mid$ & \begin{tabular}{|l|} 
Trans- \\
verse
\end{tabular} & $\mid \begin{array}{l}\text { Longi- } \\
\text { tudinal }\end{array}$ & \begin{tabular}{|c|} 
Trans: \\
verse
\end{tabular} & \begin{tabular}{|l|} 
Longi- \\
tudinal
\end{tabular} & $\begin{array}{c}\text { Trans- } \\
\text { verse }\end{array}$ & $\left|\begin{array}{l}\text { Longi- } \\
\text { tudinal }\end{array}\right|$ & \begin{tabular}{|l|} 
Tranș- \\
verse
\end{tabular} & $\left|\begin{array}{l}\text { Longi- } \\
\text { tudinal }\end{array}\right|$ & $\begin{array}{l}\text { Trans- } \\
\text { verse }\end{array}$ & \begin{tabular}{|l|} 
Longi- \\
tudinal
\end{tabular} & \begin{tabular}{|c|} 
Trans- \\
verse
\end{tabular} & $\left|\begin{array}{l}\text { Longi- } \\
\text { tudinal }\end{array}\right|$ & $\begin{array}{l}\text { Trans- } \\
\text { verse }\end{array}$ & & ingl- \\
\hline $\begin{array}{r}\text { Elongation in } 2 \text { in. or } 50 \\
\text { mm, (or } 4 D \text { ), min, } \% \text { : } \\
\text { Basic minimum elon- } \\
\text { gation for walls } 5 / 16 \\
\text { in. [ } 8 \mathrm{~mm} \text { ] and over } \\
\text { in thickness, strip } \\
\text { tests, and for all } \\
\text { small sizes tested in } \\
\text { full section }\end{array}$ & 35 & 25 & 30 & 20 & 30 & 16.5 & 30 & 16.5 & 30 & 22 & 22 & $\cdots$ & 28 & $\cdots$ & 22 & $\ldots$ & $\therefore$ & $8^{A}$ \\
\hline $\begin{array}{l}\text { When standard round } \\
2 \text {-in. or } 50 \text {-mm gage } \\
\text { length or proportion- } \\
\text { ally smaller size test } \\
\text { specimen with the } \\
\text { gage length equal to } \\
4 D \text { (4 times the di- } \\
\text { ameter) is used }\end{array}$ & 28 & 20 & 22 & 14 & 22 & 12 & 22 & 12 & 22 & 14 & 16 & $\cdots$ & $\cdots$ & $\cdots$ & 16 & $\cdots$ & 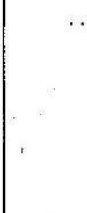 & 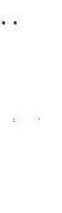 \\
\hline $\begin{array}{l}\text { For strip tests, a de- } \\
\text { duction for each } 1 / 32 \\
\text { in. }[0.8 \mathrm{~mm}] \mathrm{de}- \\
\text { crease in wall thick- } \\
\text { ness below } 5 / 16 \text { in. } \\
{[8 \text { mm from the }} \\
\text { basic minimum elon- } \\
\text { gation of the follow- } \\
\text { ing percentage }\end{array}$ & $1.75^{8}$ & $1.25^{B}$ & $1.50^{B}$ & $1.00^{B}$ & $1.50^{8}$ & $1.00^{B}$ & $1.50^{8}$ & $1.00^{B}$ & $1.50^{B}$ & $1.00^{8}$ & $1.25^{B}$ & $\cdots$ & $1.50^{B}$ & $\cdots$ & $1.25^{B}$ & $\cdots$ & 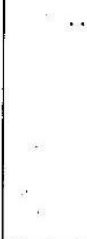 & $\begin{array}{l} \\
\therefore\end{array}$ \\
\hline & & & & & & & & & & & & & & & & & & \\
\hline
\end{tabular}

Em termos de tenacidade, a norma ASTM A333 [5] descreve os valores mínimos de energia a serem obtidos em ensaios de impacto Charpy. Estes valores estão reproduzidos na Tabela 3 para vários graus da norma, inclusive para o grau 6. As temperaturas nas quais os testes devem ser realizados estão apresentadas na Tabela 4, sendo que a temperatura exigida para os tubos em conformidade com ASTM A333[1] Gr. 6 é de $-45^{\circ} \mathrm{C}$ (ou $-50^{\circ} \mathrm{F}$ ).

A temperatura exigida para os testes do grau 6 é a menos severa dentre todos os graus especificados pela norma ASTM A333 [5]. Pode-se notar, pelos dados da Tabela 4, que os graus que obrigatoriamente necessitam de elementos de liga, conforme exigido pela norma (vide Tabela 1), devem apresentar os valores de tenacidade exigidos na Tabela $3(18 \mathrm{~J})$ em temperaturas muito mais baixas (de $-60 \mathrm{a}$ até $\left.-195^{\circ} \mathrm{C}\right)$.

O valor solicitado de energia Charpy de média mínimo ( $\left.E_{C} \geq 18 J\right)$ ou de mínimo individual $\left(E_{C} \geq 14 \mathrm{~J}\right)$ são relativamente baixos em relação aos valores de 27 a $40 \mathrm{~J}$ geralmente definido na bibliografia como sendo o mínimo aceitável para estas aplicações [8, 9 e 10].

\subsubsection{Aplicação do material informado pelo cliente}

Os tubos analisados por este trabalho são empregados no transporte de fluídos em plataforma de Petróleo a temperaturas médias de $20^{\circ} \mathrm{C}$, podendo atingir picos mínimos de $0^{\circ} \mathrm{C}$, conforme informações repassadas do projeto. Assim sendo, as temperaturas de aplicação dos tubos são normais e não exigiriam condições 
especiais para o emprego de elementos de liga adicionais aos elementos tradicionais empregados nos aços, especialmente quando se considera o baixo nível de resistência exigido para este material [5, 6 e 8].

Tabela 3. Requisitos de energia Charpy exigidos pela norma ASTM A333 [5].

\begin{tabular}{|c|c|c|c|c|c|}
\hline \multirow[t]{2}{*}{$\begin{array}{c}\text { Size of } \\
\text { Specimen, mm }\end{array}$} & \multicolumn{2}{|c|}{$\begin{array}{c}\text { Minimum Average Notched } \\
\text { Bar Impact Value of } \\
\text { Each Set of Three } \\
\text { Specimens }^{A}\end{array}$} & \multicolumn{3}{|c|}{$\begin{array}{c}\text { Minimum Notched Bar } \\
\text { Impact Value of One } \\
\text { Specimen Only of } \\
\text { a Set }{ }^{A}\end{array}$} \\
\hline & $\mathrm{ft} \cdot \mathrm{lbf}$ & $J$ & & $\mathrm{ft} \cdot \mathrm{lbf}$ & $j$ \\
\hline 10 by 10 & 13 & 18 & & 10 & 14 \\
\hline 10 by 7.5 & 10 & 14 & . & 8 & 11 \\
\hline 10 by 6.67 & 9 & 12 & & 7 & 9 \\
\hline 10 by 5 & 7 & 9 & $\therefore$ & $\because 5$ & 7 \\
\hline 10 by 3.33 & 5 & 7 & & 3 & 4 \\
\hline 10 by 2.5 & 4 & 5 & & 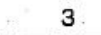 & 4 \\
\hline
\end{tabular}

A Straight line interpolation for intermediate values is permitted.

Tabela 4. Temperaturas para a realização dos ensaios de Charpy exigidos pela norma ASTM A333 [5].

\begin{tabular}{rrr}
\hline \multirow{2}{*}{ Grade } & \multicolumn{2}{c}{ Minimum Impact Test Temperature } \\
\cline { 2 - 3 } & ${ }^{\circ} \mathrm{F}$ & ${ }^{\circ} \mathrm{C}$ \\
\hline 1 & -50 & -45 \\
3 & -150 & -100 \\
4 & -150 & -100 \\
6 & -50 & -45 \\
7 & -100 & -75 \\
8 & -320 & -195 \\
9 & -100 & -75 \\
10 & -75 & -60 \\
\hline
\end{tabular}

Desta forma, pequenas quantidades de Carbono (da ordem de 0,15\%), Manganês (da ordem de 0,70\%), Silício (da ordem de 0,30\%) e Alumínio, este último presente em pequena quantidade devido ao seu uso como desoxidante conforme a norma ASTM A333 [5], seriam adequados para atender aos requisitos de resistência mecânica em tração [11] e impacto Charpy [12]. Esta condição mais do justifica o uso do grau 6 da norma ASTM A333 [5], já que o mesmo atende perfeitamente a esta aplicação, sem a necessidade de elementos de liga.

\subsection{Consumível para Soldagem}

O consumível avaliado como sendo possivelmente o mais adequado para a realização da soldagem dos tubos de aço ASTM A333 grau 6 [5], analisados neste estudo, pelo processo de soldagem TIG (GTWA), é o ER 70S3 [4]. O consumível ER $70 S 3$ [4], fornecido na forma de varetas, possui características químicas mais similares a dos tubos em questão, conforme definida pala norma ASME Sec. II Part. C [4] e seguida por fabricantes de consumíveis [13]. Trata-se de um consumível de solda que gera um metal depositado constituído de um aço de baixo carbono, não ligado, que apresenta resistência mecânica média e uma boa tenacidade (energia Charpy) em temperaturas a até $-20^{\circ} \mathrm{C}$.

Os valores da composição química regulamentada por norma estão apresentados na Tabela 4 para o consumível ER 70 S3 e na Tabela 5 para o consumível ER 80S Ni2. Já as características mecânicas exigidas pelo código ASME [4] para o consumível ER70S3 estão descritas nas Tabelas 6 e 7. 
mediu-se o potencial elétrico de pilha (galvânico) desenvolvido em um circuito elétrico fechado entre os dois consumíveis submergidos em água do mar. Este potencial elétrico foi mensurado por um multímetro Mimipa ET-3910 e serve para verificar a possibilidade de degradação galvânica entre estes dois tipos de aços.

Foram realizadas análises químicas de amostras do tubo ASTM A333 grau 6 [5] e do consumível ER70S3 [4] depositado em juntas soldadas destes tubos através de um Espectrômetro de Emissão Ótica GNR Metal Lab 75/80J para confirmar esta similaridade. Além disso, foram realizados ensaios impacto Charpy a $-45^{\circ} \mathrm{C}$ em Corpos de Prova (CPs) retirados do metal base (MB), zona termicamente afetada (ZTA) pelo calor da solda e do metal de solda (MS) de juntas de tubos ASTM A333 grau 6 [5], soldadas com consumível ER70S3 [4], fornecido pela Gerdau [13]. Além da energia Charpy, foram registradas as áreas dúcteis (ADs) das fraturas dos CPs.

Tabela 9. Características químicas e mecânicas do consumível ER $70 S 3$ conforme fornecido [13].

\begin{tabular}{|c|c|c|c|c|c|c|c|c|c|c|c|c|}
\hline \multicolumn{3}{|c|}{ BITOLAS EM mm TOL $(+0,01-0,04)$} & \multicolumn{4}{|c|}{ BITOLAS EM mm TOL $(+0,01-0,07)$} & \multicolumn{3}{|c|}{ ACONDICIONAMENTO } & $\begin{array}{c}\text { PESO } \\
(\mathrm{kg})\end{array}$ & \multicolumn{2}{|c|}{ EMBALAGEM } \\
\hline 1,59 & 2,00 & 2,38 & 3,00 & 3,18 & 3,97 & 4,76 & \multicolumn{3}{|c|}{ VARETA } & 10 & \multicolumn{2}{|c|}{ TUBO } \\
\hline CLASSE & TIPO & ELEMENTOS & c & Si & Mn & $\mathbf{s}$ & $\mathbf{P}$ & $\mathrm{Cu}$ & $\mathbf{N i}$ & $\mathrm{Cr}$ & Mo & v \\
\hline \multirow{2}{*}{ AWS A 5.18} & \multirow{2}{*}{ ER 70S-3 } & $\%$ MíN. & 0,06 & 0,45 & 0,9 & - & - & - & - & - & - & - \\
\hline & & \% MÁX. & 0,15 & 0,75 & 1,4 & 0,035 & 0,025 & $0,5^{\star}$ & 0,15 & 0,15 & 0,15 & 0,03 \\
\hline
\end{tabular}

\section{RESULTADOS E DISCUSSÃO}

A Tabela 10 apresenta as composições químicas obtidas do metal base (MB) e de solda (MS) das juntas testadas. Pode-se notar que a composição química do aço do metal de solda é bem similar ao indicado pelo fabricante [13] (Tabela 9) e compatível com a composição química do aço ASTM A333 Gr. 6 (Tabela 1). Esta condição garante uma melhor homogeneidade física e mecânica do material assim como uma boa soldabilidade entre os mesmos, pois em termos de carbono equivalente, por exemplo definido pela fórmula do International Institute of Welding (IIW): CEQ = \%C $+\% \mathrm{Mn} / 6+(\% \mathrm{Cr}+\% \mathrm{~V}+\% \mathrm{Mo}) / 5+(\% \mathrm{Ni}+\% \mathrm{Cu}) / 15$, os dois metais são praticamente iguais e apresentam um baixo carbono equivalente.

Tabela 10. Composição química da tubulação (metal base) e do metal de solda depositado a partir do consumível ER 70 S3 empregado neste trabalho.

\begin{tabular}{|c|c|c|c|c|c|c|c|}
\hline \multirow{9}{*}{ Metal Base } & Elementos & C & $\mathbf{S i}$ & Mn & $\mathbf{P}$ & $\mathbf{S}$ & $\mathrm{Cr}$ \\
\hline & Obtido & 0,203 & 0,27 & 1,25 & 0,005 & 0,003 & 0,03 \\
\hline & $* U$ & $\pm 0,012$ & $\pm 0,02$ & $\pm 0,02$ & $\pm 0,004$ & $\pm 0,004$ & $\pm 0,03$ \\
\hline & Elementos & Mo & $\mathrm{Ni}$ & Nb & Al & $\mathrm{Cu}$ & Co \\
\hline & Obtido & ND & ND & 0,008 & 0,014 & 0,040 & 0,003 \\
\hline & $* U$ & $\pm 0,03$ & $\pm 0,04$ & $\pm 0,003$ & $\pm 0,005$ & $\pm 0,01$ & $\pm 0,005$ \\
\hline & Elementos & $\mathrm{Ti}$ & V & B & $\mathbf{P b}$ & Sn & W \\
\hline & Obtido & 0,003 & 0,005 & 0,0008 & 0,023 & ND & 0,059 \\
\hline & $* U$ & $\pm 0,002$ & $\pm 0,018$ & $\pm 0,0015$ & $\pm 0,013$ & $N C$ & $\pm 0,008$ \\
\hline
\end{tabular}




\begin{tabular}{|c|c|c|c|c|c|c|c|}
\hline \multirow{9}{*}{ Metal de Solda } & Elementos & C & Si & Mn & $\mathbf{P}$ & $\mathbf{S}$ & $\mathrm{Cr}$ \\
\hline & Obtido & 0,078 & 0,45 & 1,00 & 0,013 & 0,007 & 0,02 \\
\hline & $* U$ & $\pm 0,012$ & $\pm 0,02$ & $\pm 0,02$ & $\pm 0,004$ & $\pm 0,004$ & $\pm 0,03$ \\
\hline & Elementos & Mo & $\mathrm{Ni}$ & $\mathrm{Nb}$ & Al & $\mathrm{Cu}$ & Co \\
\hline & Obtido & ND & 0,01 & 0,004 & ND & 0,047 & 0,003 \\
\hline & $* U$ & $\pm 0,03$ & $\pm 0,04$ & $\pm 0,003$ & $\pm 0,005$ & $\pm 0,01$ & $\pm 0,005$ \\
\hline & Elementos & $\mathrm{Ti}$ & V & B & $\mathbf{P b}$ & Sn & $\mathbf{W}$ \\
\hline & Obtido & 0,001 & 0,002 & 0,0006 & 0,010 & ND & 0,018 \\
\hline & $* U$ & $\pm 0,002$ & $\pm 0,018$ & $\pm 0,0015$ & $\pm 0,013$ & $N C$ & $\pm 0,008$ \\
\hline
\end{tabular}

Além da questão da soldabilidade, uma composição química similar evita a ocorrência de processos corrosivos de origem galvânica, quando dois metais ou ligas metálicas com composições químicas dissimilares são unidos formando um par galvânico. Este tipo de processo corrosivo é perigoso, pois ocorre de forma localizada.

Para fazer uma análise comparativa, verificou-se o potencial entre os metais analisados. Neste caso verificou-se que entre os dois consumíveis (ER 70S3 e ER $80 \mathrm{~S} \mathrm{Ni2}$ ) houver a geração de um potencial de $0,2 \mathrm{~V}$ no meio água marinha. Esse foi o mesmo valor registrado entre o metal do tubo A333 Gr. 6 e consumível ER 80S Ni2. Trata-se de um valor baixo, mas suficiente para produzir degradação galvânica ao longo do tempo de uso planejado da tubulação.

Em termos de tenacidade, a maior diferença entre o aço ASTM A333 Gr. 6 (Tabela 3) e o consumível ER $70 \mathrm{~S} 3$ (Tabela 7 ) é a temperatura de garantia; $-45^{\circ} \mathrm{C}$ para 0 tubo de aço e $-20^{\circ} \mathrm{C}$ para o consumível; e o valor de energia a ser garantido; $18 \mathrm{~J}$ para o tubo de aço e 27J para o consumível. Como as temperaturas de teste são diferentes, não é possível fazer uma comparação direta entre os dois aços. Porém em termos de garantia absoluta é possível afirmar que o consumível ER $70 S 3$ é perfeitamente compatível com o tubo de aço ASTM A333 Gr. 6 na temperatura especifica de uso da tubulação $\left(0^{\circ} \mathrm{C}\right)$.

A Tabela 11 apresenta os dados obtidos nos ensaios Charpy e a Figura 1 apresenta o aspecto dos CPs após a ruptura no teste Charpy a $-45^{\circ} \mathrm{C}$. Nota-se que o valor da tenacidade a impacto Charpy foi bem mais elevada do que a registrada pelo metal base. O que pode ser atribuído ao baixo teor dos elementos de liga presentes no metal de solda, especialmente ao teor de carbono, conforme mostrado na Tabela 10.

Tabela 11. Resultados dos testes de impacto Charpy a $-45^{\circ} \mathrm{C}$ da tubulação (metal base) e do consumível ER 70 S3 empregado no trabalho (metal de solda).

\begin{tabular}{cccc}
\hline & Metal de Solda & ZTA & Metal Base \\
\hline Energia Média $(\mathrm{J})$ & 143 & 108 & 43 \\
\%Área Dúctil & $100 \%$ & $65 \%$ & $22 \%$ \\
\hline
\end{tabular}
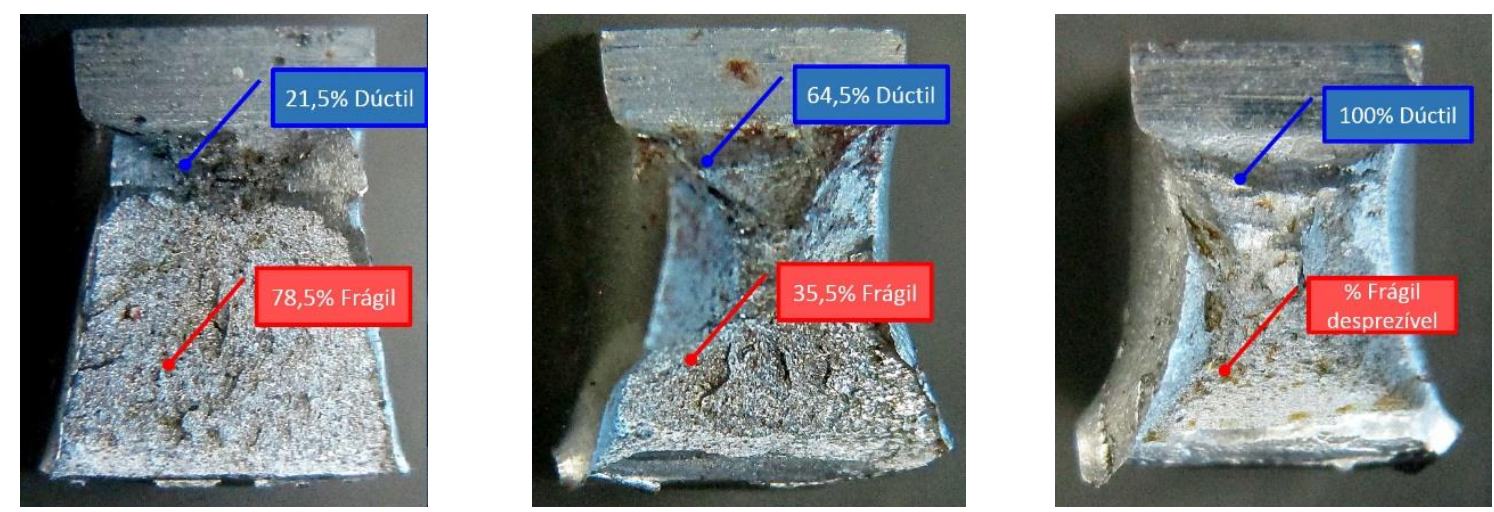
METAL BASE

ZTA

Figura 1. Superfícies de fratura obtidas no teste de impacto Charpy.

METAL DE SOLDA

Além das considerações feitas, a norma ASME B31.3[14] informa, em sua tabela A-1 as características do metal da norma ASTM A333 Gr. 6 [5] que são relevantes aos trabalhos de solda. Esta tabela apresenta a uma nota técnica, que cita uma nova tabela nesta norma (Table 323.2.2) que isenta a junta solda de testes de impacto Charpy no processo de qualificação do procedimento de solda, caso o material seja empregado, em projeto, em temperaturas acima de $-29^{\circ} \mathrm{C}$.

\section{CONCLUSÃO}

O uso do consumível ER $70 S 3$ é viável para a soldagem de tubulações de aço ASTM A333 Gr.6, se os mesmos forem empregados em temperaturas de trabalho até um mínimo de $-29^{\circ} \mathrm{C}$, conforme resultados obtidos e conforme a ASME B31.1. Nestes casos, o consumível ER 70S3 não é só permitido pelas normas técnicas vigentes, como também é preferível em termos metalúrgicos e químicos por garantir melhor homogeneidade mecânica e por evitar eventuais efeitos de corrosão galvânica. Novos estudos envolvendo ensaios de corrosão acelerada de amostras de chapas e tudos com estas juntas foram realizados e serão publicados em outra contribuição técnica para neste evento.

\section{REFERÊNCIAS}

1 LANCASTER, J. Handbook of structural welding. Cambridge (UK): Abington Hall, 1997. Cap. 2, p. 56-113.

2 MORAIS, W. A.; MAGNABOSCO, A. S; NETTO, E. B. M. Metalurgia física e mecânica aplicada. 2. ed. São Paulo: ABM, 2009.

3 MODENESI, Paulo J.; MARQUES, Paulo Villani; BRACARENSE, Alexandre Q. Soldagem Fundamentos e Tecnologia. Universidade Federal de Minas Gerais. Departamento de Engenharia Metalúrgica. Belo Horizonte, 2007.

4 ASME. ASME Sec. II, Boiler and Pressure Vessel Code. Part C: Specifications for Welding Rods, Electrodes, and Filler Metals, 2010

5 AMERICAN SOCIETY FOR TESTING AND MATERIALS. ASTM A333/A333M:13. Standard Specification for Seamless and Welded Steel Pipe for Low-Temperature Service and Other Applications with Required Notch Toughness, 2013.

6 GUIA DA SIDERUGIA BRASILEIRA E METAIS NÃO FERROSOS. Disponível em: http://www.guiadasiderurgia.com.br/novogs/Flip/GC_2014/index.html\#120<Acesso em 05/02/2015>. Grip Editora, 2014, p.120-122.

7 AMERICAN SOCIETY FOR TESTING AND MATERIALS. ASTM A1040:04. Standard Guide for Specifying Harmonized Standard Grade Compositions for Wrought Carbon, Low-Alloy, and Alloy Steels, 2004.

8 AMERICAN PETROLEUM INSTITUTE. Specification for Line Pipe, API Specification 5L, 44th edition, March, 2007.

9 AMERICAN PETROLEUM INSTITUTE. Specification for Steel Plates for Offshore Structures Produced by Thermo-Mechanical Control Processing (TMCP), API Specification 2W, 5th edition, March, 2007.

10 AMERICAN SOCIETY FOR TESTING AND MATERIALS. ASTM A131/A131M:14. Standard Specification for Structural Steel for Ships, 2014.

11 COMITRE, T. B.; BUENO, R. D.; BLEY, R. F.; SPAGNUOLO, E. S.; ESCOBAR, A. A.; NASCIMENTO, F. C. ; ALVES, M. B. ; OLIVEIRA, D. S. ; MORAIS, W. A. Equacionamento e quantificação das propriedades mecânicas de barras de aço em conformidade com normas SAE. Siderurgia Brasil, v. 13, p. 32-38, 2012. (planilha e artigo disponíveis em:

http://www.inda.org.br/interna.php?pagina=propriedade_mecanica <Acesso em 05/02/2015>). 
12 ASM INTERNATIONAL. ASM Handbook Volume 01: Properties and Selection: Irons, Steels, and High-Performance Alloys, vol. 01, Materials Park, 1990.

13 GERDAU. Arames para solda. Disponível em:

https://www.comercialgerdau.com.br/produtos/download/12_AramesSolda.pdf $<$ Acesso em 05/02/2015>.

14 ASME. ASME B31.3. Process Piping - ASME Code for Pressure Piping, B31, 2010. 